\title{
Spin-orbit-assisted electron pairing in one-dimensional waveguides
}

\author{
François Damanet $\odot,{ }^{1,2,3, *}$ Elliott Mansfield, ${ }^{1,2}$ Megan Briggeman, ${ }^{2,4}$ Patrick Irvin, ${ }^{2,4}$ Jeremy Levy $\odot,{ }^{2,4}$ \\ and Andrew J. Daley ${ }^{1,2}$ \\ ${ }^{1}$ Department of Physics and SUPA, University of Strathclyde, Glasgow G4 ONG, Scotland, United Kingdom \\ ${ }^{2}$ Pittsburgh Quantum Institute, Pittsburgh, Pennsylvania 15260, USA \\ ${ }^{3}$ Institut de Physique Nucléaire, Atomique et de Spectroscopie, CESAM, University of Liège, B-4000 Liège, Belgium \\ ${ }^{4}$ Department of Physics and Astronomy, University of Pittsburgh, Pittsburgh, Pennsylvania 15260, USA
}

(Received 21 December 2020; revised 21 May 2021; accepted 29 June 2021; published 1 September 2021)

\begin{abstract}
Understanding and controlling the transport properties of interacting fermions is a key forefront in quantum physics across a variety of experimental platforms. Motivated by recent experiments in one-dimensional (1D) electron channels written on the $\mathrm{LaAlO}_{3} / \mathrm{SrTiO}_{3}$ interface, we analyze how the presence of different forms of spin-orbit coupling (SOC) can enhance electron pairing in 1D waveguides. We first show how the intrinsic Rashba SOC felt by electrons at interfaces such as $\mathrm{LaAlO}_{3} / \mathrm{SrTiO}_{3}$ can be reduced when they are confined in one dimension. Then, we discuss how SOC can be engineered, and show using a mean-field Hartree-FockBogoliubov model that SOC can generate and enhance spin-singlet and -triplet electron pairing. Our results are consistent with two recent sets of experiments [Briggeman et al., Nat. Phys. 17, 782787 (2021); Sci. Adv. 6, eaba6337 (2020)] that are believed to engineer the forms of SOC investigated in this work, which suggests that metal-oxide heterostructures constitute attractive platforms to control the collective spin of electron bound states. However, our findings could also be applied to other experimental platforms involving spinful fermions with attractive interactions, such as cold atoms.
\end{abstract}

DOI: 10.1103/PhysRevB.104.125103

\section{INTRODUCTION}

There is a fundamental interest in investigating transport in reduced-dimensionality systems, where interactions play a prominent role and can lead to exotic phases of matter [1]. Understanding transport dynamics in these systems and how they can be controlled is also crucial to shed light on the properties and the further development of useful materials.

In the solid state, different platforms have been developed to offer playground for the investigation of strongly correlated systems. Of particular interest for this work are the metal-oxide heterostructures, such as the $\mathrm{LaAlO}_{3}$ and $\mathrm{SrTiO}_{3}$ interface (LAO/STO) [2,3]. By depositing just a few layers of LAO onto the bulk STO substrate, a two-dimensional electron gas (2DEG) can be formed at the interface. This 2DEG is highly controllable, and can be engineered to display many interesting phenomena, such as superconductivity [4], tuneable transport [5], Rashba spin-orbit coupling [6,7], or ferromagnetic phases [8]. Notably, using conductive atomicforce microscope (c-AFM) lithography, it has been shown that nanostructures at the LAO/STO interface can be created, allowing in particular for the exploration of quantum transport of electrons with tunable attractive interactions [9] in engineered quasi-1D nanowires [10]. This method has led to the observation of quantized ballistic transport of single and paired electrons [11], as well as more exotic bound states of three or more electrons [12]. The AFM tip used to create the

*fdamanet@uliege.be nanostructures acts as a nanoscale pencil that can reversibly tune the transport properties of the interface from insulating to conducting via (de)protonation $[13,14]$, and offers great potential to study and engineer new transport phenomena and new phases of quantum matter. The level of control being developed here evokes comparison with the high control of quantum simulators in other platforms [15,16], such as cold atoms [17], coupled light resonator arrays [18], trapped ions [19], superconducting circuits [20], or Rydberg atoms [21].

Driven by the long-term prospect of developing analog quantum simulators in the solid state, more recent experiments in LAO/STO interfaces have shown that by spatially modulating the AFM tip during the writing process, it is possible to create laterally undulating wires [22] and 1D KronigPenney-like superlattice structures [23]. Compared to straight waveguides [11], these devices exhibit stable fractional conductance plateaux and enhanced pairing of electrons. A possible explanation for some of the observed phenomena is that the modulation engineers a spin-orbit coupling (SOC) in the waveguide which modifies its transport properties. SOC constitutes a useful resource to manipulate spins in a wide range of applications and has led to the discovery of new topological classes of materials [24]. SOC appears naturally in crystals that lack an inversion symmetry, such as the LAO/STO interface, but could also be engineered artificially by creating effective broken inversion symmetries via applied electric fields.

The goal of this theoretical work is to analyze how the presence of spin-orbit interactions can enhance the pairing of electrons in 1D waveguides such as the ones realized on 
(a)

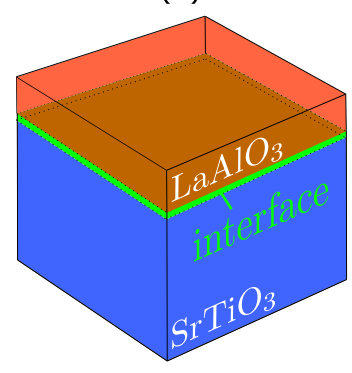

(b)

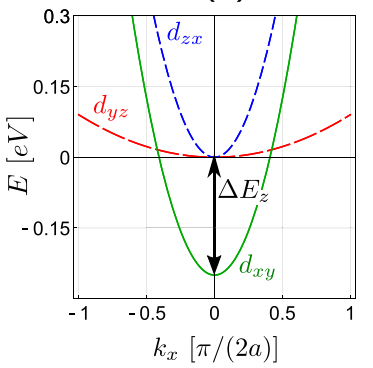

(c)

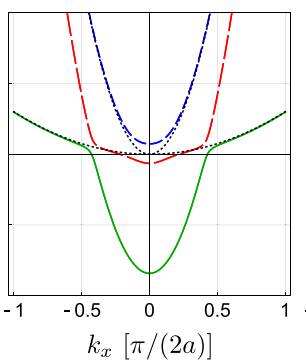

(d)

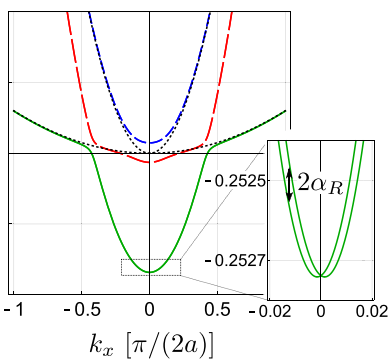

(e)

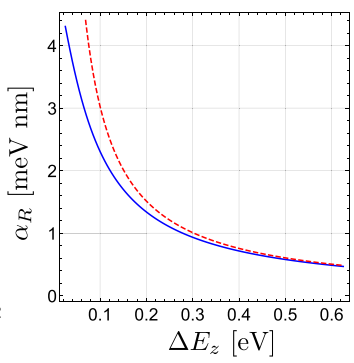

FIG. 1. Electrons at the 2D LAO/STO interface. (a) Diagram of a typical LAO/STO interface (green) between a few layers of LaAlO 3 (red) on top of bulk $\mathrm{SrTiO}_{3}$ (blue). (b) Energies of $H_{0}$ [Eq. (1)] as a function of $k_{x} \in[-\pi /(2 a), \pi /(2 a)]$ where $a=0.392 \mathrm{~nm}$ is the lattice spacing. The parameters are $m_{h}=6.8 m_{e}, m_{l}=0.41 m_{e}, \Delta E_{z}=0.25 \mathrm{eV}$, where $m_{e}$ is the electron mass. The spin-degenerate orbital $d_{x y}$ (green solid line) is the lowest energy state due to the confinement energy $\Delta E_{z}$ along $z$, compared to $d_{y z}$ (red long-dashed line) and $d_{z x}$ (blue short-dashed line). (c) Energies of $H_{0}+H_{\text {aso }}$ as a function of $k_{x}$, for $\Delta_{\text {aso }}=19.3 \mathrm{meV}$ and other parameters as above. The atomic spin-orbit coupling Hamiltonian $H_{\text {aso }}$ [Eq. (2)] mixes the orbitals at the crossing points, so does not affect much the low-energy electrons in the bottom of the lowest orbital $d_{x y}$ (near $k_{x} \approx 0$ ). The dotted black lines correspond to the energies of $H_{0}$ as in Fig. 1(b) for comparison. (d) Energies of the full Hamiltonian $H_{\mathrm{tot}}=H_{0}+H_{\mathrm{aso}}+H_{a}$ [Eq. (4)] as a function of $k_{x}$, for $\Delta_{z}=20 \mathrm{meV}$ and other parameters as above. The Hamiltonian $H_{a}$ lifts the spin degeneracy and generates a linear Rashba SOC of strength $\alpha_{R}$ for the electrons in the lowest band, as highlighted in the inset. (e) Rashba SOC strength as a function of $\Delta E_{z}$ obtained from the numerical diagonalization of $H$ [Eq. (4)] (blue solid line) and from the perturbative theory [Eq. (6)] (dashed red line)], for other parameters as above [38]. The Rashba SOC decreases for increasing confinement along $z$.

the LAO/STO interface, elaborating on the theory discussed in [23]. Our work suggests that heterostructures constitute an attractive platform for controlling collective spin states of electron pairs. However, the theory presented here is quite general and could be used to describe other experimental platforms involving 1D systems of spinful fermionic particles with attractive interactions and SOC, such as cold atoms $[25,26]$. SOC can indeed be induced in these systems via the use of artificial gauge fields [27-30], and the interplay between SOC and interactions is starting to be explored with cold atoms [31-35], showing notably the possibility to enhance pairing via SOC. Our findings are consistent with these results but in different forms and parameter regimes.

This paper is structured as follows. In Sec. II, we review the band model typically used to describe the origin of the intrinsic Rashba spin-orbit coupling that can exist in crystals that lack inversion symmetry, such as the LAO/STO interface, and provide some arguments on how it can be reduced when the electrons are confined in a 1D channel. We then elaborate on ways to engineer different forms of SOC in the waveguide. In Sec. III, we derive a single-particle model for these electrons that includes the SOC and the electronelectron interactions at the mean-field level. In Sec. IV, we solve our model to study the interplay between interactions and SOC, showing that the SOC can enhance spin-singlet and spin-triplet pairing. In Sec. V, we finally conclude and present some perspectives of our work.

\section{ELECTRONS AT INTERFACES WITH SOC}

In this section, we first present a review of a simple band model for electrons at heterostructure interfaces that explains the origin of the Rashba spin-orbit coupling, following [36,37]. Then, elaborating on the model presented in [37], we show how it can be reduced when the electrons are confined in a 1D channel. Finally, we discuss ways to engineer different forms of SOCs in such systems.

For concreteness and clarity, the theory presented below is based on the electronic structure of the LAO/STO interface. However, the theory is quite general, and could be applied to other interfaces by repeating the procedure below with modified parameters and initial electron orbitals, depending on where the Fermi energy lies in the relevant material.

\section{A. Band structure of electrons at an interface with SOC: A short review for LAO/STO}

Figure 1(a) presents a sketch of a typical LAO/STO structure, where a few layers of $\mathrm{LaAlO}_{3}$ are placed on the top of bulk $\mathrm{SrTiO}_{3}$. In STO-based interfaces, the Fermi energy lies in the $3 d t_{2 g}$ orbitals $d_{y z}, d_{x z}$, and $d_{x y}$ of the Ti ions near the interface. The electrons are naturally confined in the direction normal to it (labeled as the $z$ direction) to form a two-dimensional electron gas [green layer in Fig. 1(a)]. The backbone of the electron dynamics can be accounted for by a simple six-dimensional Hamiltonian $H_{0}$, which, in the orbital and spin basis $\left(d_{y z}, d_{x z}, d_{x y}\right) \otimes(\uparrow, \downarrow)$, takes the form $[37,39,40]$,

$H_{0}=\left(\begin{array}{ccc}\frac{\hbar^{2} k_{x}^{2}}{2 m_{h}}+\frac{\hbar^{2} k_{y}^{2}}{2 m_{l}} & 0 & 0 \\ 0 & \frac{\hbar^{2} k_{x}^{2}}{2 m_{l}}+\frac{\hbar^{2} k_{y}^{2}}{2 m_{h}} & 0 \\ 0 & 0 & \frac{\hbar^{2} k_{x}^{2}}{2 m_{l}}+\frac{\hbar^{2} k_{y}^{2}}{2 m_{l}}-\Delta E_{z}\end{array}\right) \otimes \mathbb{1}_{2}$,

where $m_{h}$ and $m_{l}$ are effective heavy and light masses, $\Delta E_{z}$ is the energy splitting due to the natural confinement of the bands along $z$-making $d_{x y}$ the lowest band at small $k$ values-and $\mathbb{1}_{2}$ is the identity operator acting on the 
two-dimensional spin Hilbert space. The Hamiltonian $H_{0}$ is naturally diagonal in the orbital basis and spin degenerate. Figure 1(b) shows its energies as a function of $k_{x}$ for reasonable parameter values.

The Hamiltonian $H_{0}$ is too simple to capture all the interesting features of the electron gas. In order to account for the effect of the atomic spin-orbit coupling, the Hamiltonian,

$$
H_{\text {aso }} \propto \mathbf{L} \cdot \boldsymbol{\sigma}=i \Delta_{\text {ASO }}\left(\begin{array}{ccc}
0 & \sigma_{z} & -\sigma_{y} \\
-\sigma_{z} & 0 & \sigma_{x} \\
\sigma_{y} & -\sigma_{x} & 0
\end{array}\right),
$$

is added to $H_{0}$, where $\mathbf{L}=\mathbf{r} \times \mathbf{p}$ is the orbital momentum operator, $\boldsymbol{\sigma}=\left(\sigma_{x}, \sigma_{y}, \sigma_{z}\right)$ is the vector of Pauli operators, and $\Delta_{\text {aso }}$ is the atomic spin-orbit coupling strength. Figure 1(c) shows the energies of $H_{0}+H_{\text {aso }}$ as a function of $k_{x}$, where $H_{\text {aso }}$ has the effect to mix the eigenstates of $H_{0}$. We chose $\Delta_{\text {aso }}=19.3 \mathrm{meV}$ as in [36,39], motivated by the fact that it leads to the same modifications of the orbitals as the ones obtained via a more refined calculation of the band structure via density functional theory (DFT) [39].

Finally, due to the broken inversion symmetry at the interface along $z$, an additional coupling of the orbital $d_{x y}$ to $d_{y z}$ and $d_{x z}$ appears, at the origin of a Rashba spin-orbit coupling, as discussed below. This effect can be accounted for via a third Hamiltonian of the form $[36,37,41,42]$,

$$
H_{a}=i \Delta_{z} a\left(\begin{array}{ccc}
0 & 0 & k_{x} \\
0 & 0 & k_{y} \\
-k_{x} & -k_{y} & 0
\end{array}\right) \otimes \mathbb{1}_{2},
$$

where $a=0.392 \mathrm{~nm}$ is the lattice spacing and $\Delta_{z}$ is the overall energy scale. Figure 1(d) shows the eigenvalues of the full Hamiltonian,

$$
H_{\text {tot }}=H_{0}+H_{\text {aso }}+H_{a},
$$

using $\Delta_{z}=20 \mathrm{meV}$, as can be again extracted from DFT [36]. As can be seen in the inset of the figure, $H_{a}$ causes a lifting of the spin degeneracy of the two lowest energies. For small $k$, this energy splitting has the form of a linear Rashba term,

$$
\Delta E_{R}=\alpha_{R}\left(k_{x} \pm i k_{y}\right)
$$

with $\alpha_{R}$ being the spin-orbit coupling strength (with dimensions of energy $\times$ length). The value of $\alpha_{R}$ can be obtained from the model by simply fitting the difference between the two lowest energies of $H_{\text {tot }}=H_{0}+H_{\text {aso }}+H_{a}$ at small momentum $\mathbf{k}$ by a linear function of $k_{x}$ (or $k_{y}$ ); the slope of it provides $2 \alpha_{R}$. Alternatively, one can write $H_{a}$ in the basis of the eigenstates of $H_{0}(\mathbf{k}=0)+H_{\text {aso }}$ and extract $\alpha_{R}$ from its matrix form, after identifying the offdiagonal elements corresponding to the coupling between the two lowest bands. Finally, since typically we have $\Delta E_{z} \gg$ $\Delta_{z}, \Delta_{\text {aso }}$, one can use a second-order perturbation theory (first order in $H_{\text {aso }}$ and $H_{a}$ ) to obtain the following analytical (a)

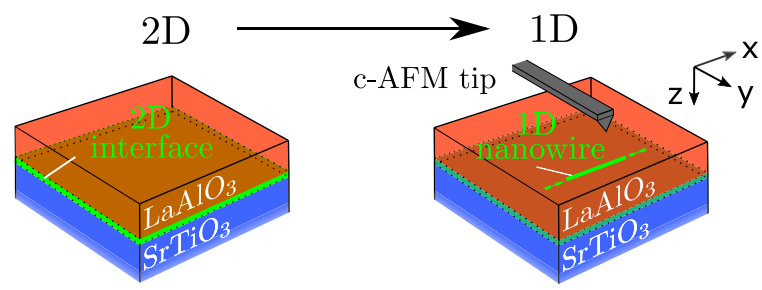

(b)
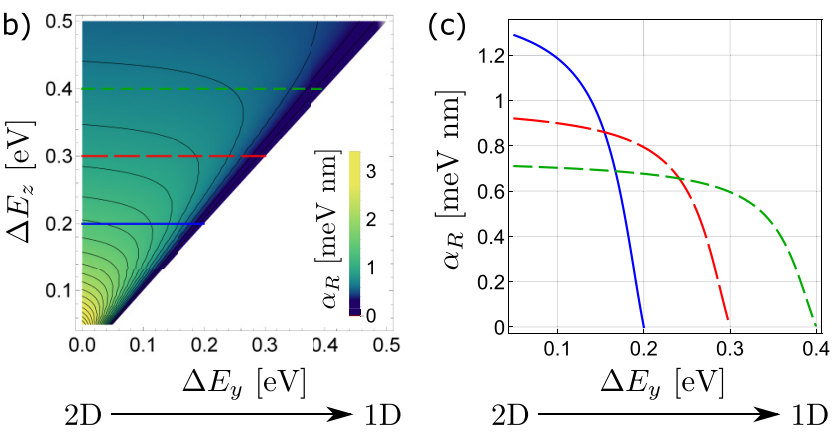

FIG. 2. Rashba SOC from two dimensions to one dimension. (a) Sketch of the transition from a $2 \mathrm{D}$ conducting interface to a 1D nanowire as realized via c-AFM lithography. (b) Rashba SOC strength $\alpha_{R}$ as a function of the lateral and vertical confinements $\Delta E_{y}$ and $\Delta E_{z}$. The values of $\alpha_{R}$ along the horizontal lines (corresponding to three specific values of $\Delta E_{z}$ ) are shown more clearly in (c). (c) Rashba SOC strength $\alpha_{R}$ as a function of the confinement $\Delta E_{y}$ for $\Delta E_{z}=0.2 \mathrm{eV}$ (solid blue line), $0.3 \mathrm{eV}$ (red long-dashed line), and $0.4 \mathrm{eV}$ (green short-dashed line). Increasing the confinement along $\Delta E_{y}$ reduces the dimensionality of the system and the Rashba SOC.

expression $[36,37]$ :

$$
\alpha_{R}=2 a \frac{\Delta_{z} \Delta_{\text {aso }}}{\Delta E_{z}}
$$

valid for $\Delta E_{z} \gg \Delta_{z}, \Delta_{\text {aso }}$. Experimentally, the value $\alpha_{R}$ is usually found to be around $1-5 \mathrm{meV} \mathrm{nm} \mathrm{[6,7],} \mathrm{and} \mathrm{the} \mathrm{model}$ presented above predicts compatible values of $\alpha_{R}$. Figure 1(e) shows $\alpha_{R}$ obtained from both the numerical diagonalization of $H_{\text {tot }}$ and the perturbative theory (6) as a function of the confinement energy $\Delta E_{z}$ and for other parameters taken from [36]. As can be seen in Fig. 1(e), increasing the confinement along $z$ reduces the linear Rashba spin-orbit coupling felt by the low-energy electrons located at the bottom of the two lowest bands.

\section{B. Effects of confinement in one dimension}

We saw in the previous section that the vertical confinement of the electrons can be accounted for by a phenomenological parameter $\Delta E_{z}$ in $H_{0}$ [Eq. (1)]. As suggested in [37], it is reasonable to think that the lateral confinement felt by the electrons in a 1D waveguide [as can be realized via c-AFM lithography as sketched in Fig. 2(a)] could be modeled in a similar way. Labelling $x$ as the direction 
of the waveguide, the modified $H_{0}$ reads [37]

$$
H_{0}^{\prime}=\left(\begin{array}{ccc}
\frac{\hbar^{2} k_{x}^{2}}{2 m_{h}}+\frac{\hbar^{2} k_{y}^{2}}{2 m_{l}} & 0 & 0 \\
0 & \frac{\hbar^{2} k_{x}^{2}}{2 m_{l}}+\frac{\hbar^{2} k_{y}^{2}}{2 m_{h}}-\Delta E_{y} & 0 \\
0 & 0 & \frac{\hbar^{2} k_{x}^{2}}{2 m_{l}}+\frac{\hbar^{2} k_{y}^{2}}{2 m_{l}}-\Delta E_{z}
\end{array}\right) \otimes \mathbb{1}_{2},
$$

where $\Delta E_{y}$ is the engineered confinement along $y$. The presence of $\Delta E_{y}$ lifts the degeneracy between the $d_{x z}$ and the $d_{y z}$ bands.

By diagonalizing

$$
H_{\mathrm{tot}}^{\prime}=H_{0}^{\prime}+H_{\mathrm{aso}}+H_{a},
$$

and then extracting numerically the value of the Rashba spinorbit coupling strength $\alpha_{R}$ along the direction of the nanowire $k_{x}$ as explained before, we can show that $\alpha_{R}$ decreases monotonically as a function of $\Delta E_{y}$ and goes to zero for $\Delta E_{y}=$ $\Delta E_{z}$, when the bands $d_{x z}$ and $d_{x y}$ become degenerate. This can be seen in Fig. 2(b), showing $\alpha_{R}$ as a function of both $\Delta E_{z}$ and $\Delta E_{y}$ for a wide range of reasonable values with $\Delta E_{y} \leqslant \Delta E_{z}$ so that the orbital $d_{x y}$ remains the ground state, degenerate with $d_{z x}$ only when $\Delta E_{y}=\Delta E_{z}$. Figure 2(c) shows more clearly three line cuts of Fig. 2(b), i.e., $\alpha_{R}$ as a function of $\Delta E_{y}$ for $\Delta E_{z}=0.2,0.3$, and $0.4 \mathrm{eV}$. While precise values of $\Delta E_{y}$ and $\Delta E_{z}$ are difficult to estimate, this result suggests that in quasi-1D nanowires, the intrinsic Rashba spin-orbit coupling due to the broken vertical inversion symmetry could be significantly smaller than in the $2 \mathrm{D}$ case (i.e., when $\Delta E_{y}=0$ ). Let us emphasize again that the procedure described here to study the impact of the confinement is quite general and can thus be adapted to other materials. Note finally that other approaches to study the effects of confinement on the spectrum of spinorbit-coupled particles can be found in [43].

\section{Engineering spin-orbit couplings}

The model presented in the previous section suggests that the usual Rashba SOC at interfaces with broken inversion symmetry could be smaller when the electrons are confined in quasi-1D nanostructures. We discuss here ways to recover a significant value of Rashba SOC, as well as other forms of SOC, before studying its effects on the transport properties of the electrons.

As mentioned in the introduction, recent transport experiments in a 1D modulated waveguide have shown signatures of SOC, both directly [22] and potentially through observation of spin-orbit enhanced electron pairing [23], which we present a description of in Sec. IV. The qualitative argument is the following: Assume the electrons are confined along $x$. When they travel through the waveguide with a velocity $\mathbf{v}=v_{x} \mathbf{e}_{x}$ where $\mathbf{e}_{i}$ is a unit vector along $i=x, y, z$, they feel an electric field produced by the modulation. For a vertical modulation as in [23], it would have the form $\mathbf{E}_{\text {eff }} \propto E_{\text {eff }}(x) \mathbf{e}_{z}$. This would lead to an effective magnetic field $\mathbf{B}_{\text {so }} \propto \mathbf{v} \times \mathbf{E}_{\text {eff }}$ along $y$ and then produce an energy shift of the form $\propto \sigma \cdot \mathbf{B}_{\mathrm{so}}$. By contrast, a lateral modulation as in [22] would be associated with an electric field $\mathbf{E}_{\text {eff }} \propto E_{\text {eff }}(x) \mathbf{e}_{y}$ and thus a magnetic field along $z$. This reasoning is of course valid for applied external fields that are not necessarily position dependent. This is at the root of the control of the Rashba SOC at the LAO/STO interface $[6,7]$.

Hence, despite the fact that the confinement along $y$ can drastically reduce the Rashba SOC at interfaces with natural broken inversion symmetry, it is possible to engineer it in different forms depending on the direction of applied electric fields, resulting, e.g., from the design of side-gate voltages in specific configurations. It is thus experimentally relevant to explore the effects of SOC on the transport dynamics of electrons, which is the purpose of the next sections.

\section{MODEL FOR ELECTRON TRANSPORT IN 1D WAVEGUIDES WITH SOC}

Having looked at how to control artificial SOC in electron waveguides, in this section, we now present a model to describe the interplay between interactions and different forms of SOC on the transport properties of the electrons. We present successively the single-particle basis of our model (Sec. III A), the electron-electron interactions we consider (Sec. III B), and finally how to solve the relevant equations of motion (Sec. III C), by means of a self-consistent mean-field model.

\section{A. Single-particle model with SOC}

We assume the electrons are confined in a 1D channel along $x$, as depicted, e.g., in Fig. 1(a), and that an external out-of-plane magnetic field $B$ is applied along $z$, introducing a Landau quantization effect [44]. The waveguide (typically $\sim 50-1000$-nm long in LAO/STO devices) is connected at both ends to unbiased leads that act as reservoirs (not shown). We consider the case where the Fermi energy of the electrons in the waveguide is tunable via a gate voltage but remains close to the bottom of the lowest band of $H_{\text {tot }}^{\prime}$ [Eq. (8)], so that the electrons have too-low momenta $k_{x}$ to populate other bands. For LAO/STO interfaces, this is motivated by the fact that in conductive nanostructures created via c-AFM lithography, the typical carrier density is around $n \sim 0.5$ $1 \times 10^{13} \mathrm{~cm}^{-2}$ [13]. Roughly speaking this would mean an associated momentum $k=\sqrt{2 \pi n} \sim 0.7-1 \times 10^{-6}(\pi / a)$, i.e., far from the crossing point between the $d_{x y}$ and $d_{y z}$ orbitals [around $\sim 0.25(\pi / a)$ in Fig. 1(b) $-1(\mathrm{~d})]$. Hence, all the transport channels are assumed to originate from a single band.

We generalize the single-particle model described in detail in [11] to include spin-orbit couplings. The Hamiltonian for spin-up and -down electrons in Landau gauge where the 
vector potential reads $\mathbf{A}=(-B y, 0,0)$ takes the form,

$$
\begin{aligned}
H= & \frac{\left(p_{x}-e B y\right)^{2}}{2 m_{x}}+\frac{p_{y}^{2}}{2 m_{y}}+\frac{p_{z}^{2}}{2 m_{z}}+V(y)+V(z)-\mu \\
& -g \frac{\mu_{B}}{2} B \sigma_{z}+\frac{\left(\alpha_{v} \sigma_{y}+\alpha_{l} \sigma_{z}\right)}{\hbar}\left(p_{x}-e B y\right) .
\end{aligned}
$$

The first line describes the (spin-degenerate) kinetic and potential energies of the electrons, where $p_{i}$ and $m_{i}(i=x, y, z)$ are the electron momentum operator and effective mass components along the different directions, where

$$
V(y)=\frac{m_{y} \omega_{y}^{2} y^{2}}{2}, \quad V(z)= \begin{cases}\frac{m_{z} \omega_{z}^{2} z^{2}}{2} & \text { for } z \geqslant 0 \\ +\infty & \text { for } z<0,\end{cases}
$$

are parabolic and half-parabolic potentials describing the transverse confinement along $y$ and $z$, with $\omega_{y}$ and $\omega_{z}$ the trapping frequencies, and where $\mu$ is the chemical potential. Here, we consider indeed that the electron cannot penetrate into one of the layers (as it is the case for the LAO layer in $\mathrm{LAO} / \mathrm{STO}$ interfaces), i.e., the confining potential along $z$ is infinite for $z<0$. The second line of Eq. (9) corresponds to the Zeeman energy term due to the out-of-plane magnetic field $B$, with $g$ the Landé factor and $\mu_{B}$ the Bohr magneton, and the last two terms are effective SOC terms of strength $\alpha_{v}$ and $\alpha_{l}$. As explained in the previous section, these two terms could be engineered through applications of electric fields vertically $(v)$ or laterally $(l)$, and thus referred to as vertically induced or laterally induced SOC. Note that in the case of modulated electric fields, the SOCs should in principle be spatially dependent, which would drastically complicate the model. In this specific scenario, we make the approximation that $\alpha_{v}$ and $\alpha_{l}$ describe the root-mean-square values of the varying parameters. Note finally that compared to the single-particle model presented in [23], our model is slightly more refined (as the vertically induced SOC reads $\propto \alpha_{v} \sigma_{y}\left(p_{x}-e B y\right)$ instead of $\left.\propto \alpha_{v} \sigma_{y} p_{x}\right)$ and is more general as it includes also laterally induced SOC.

Since the Hamiltonian (9) is translationally invariant along $x$, we can make the replacement $p_{x} \rightarrow \hbar k_{x} \equiv \hbar k$. The $x$ and $y$ motional part of Eq. (9) can then be decoupled using

$$
\begin{aligned}
& \frac{(\hbar k-e B y)^{2}}{2 m_{x}}+V(y)+\frac{\left(\alpha_{v} \sigma_{y}+\alpha_{l} \sigma_{z}\right)}{\hbar}(\hbar k-e B y) \\
& =\frac{\hbar^{2} k^{2}}{2 m_{x}} \frac{\omega_{y}^{2}}{\Omega^{2}}+\frac{m_{y} \Omega^{2}}{2}\left(y-y_{0}(k)\right)^{2}-\frac{e^{2} B^{2}}{2 m_{y} \Omega^{2} \hbar^{2}}\left(\alpha_{l}^{2}+\alpha_{v}^{2}\right) \\
& \quad+\frac{\omega_{y}^{2}}{\Omega^{2}}\left(\alpha_{v} \sigma_{y}+\alpha_{l} \sigma_{z}\right) k,
\end{aligned}
$$

where

$$
\begin{gathered}
\omega_{c}=\frac{e B}{\sqrt{m_{x} m_{y}}}, \\
\Omega=\sqrt{\omega_{y}^{2}+\omega_{c}^{2}}, \\
y_{0}(k)=\frac{\hbar \omega_{c} k}{\sqrt{m_{y} m_{x}} \Omega^{2}}+\frac{e B}{\hbar m_{y} \Omega^{2}}\left(\alpha_{v} \sigma_{y}+\alpha_{l} \sigma_{z}\right),
\end{gathered}
$$

are, respectively, the cyclotron frequency, the effective frequency, and the momentum-and-spin-dependent center of the lateral trapping. If the second term of Eq. (14) was not present, then the eigenstates of the motional part of (9) would correspond to plane waves $|k\rangle$ along $x$ and 2D harmonic oscillator eigenstates $|m, n\rangle$ along $y$ and $z$, where $m, n \in \mathbb{N}$ are the quantum numbers labeling them. In this case, the position $y_{0}(k)$ of the center-of-mass of the harmonic oscillator eigenstate along $y$ depends on the momentum $k$ of the electron along $x$. However, due to the last term of Eq. (14), this centerof-mass position is in principle also spin dependent, which complicates this picture. In Appendix A, we show, however, that for reasonable values of the parameters, the second term of Eq. (14) can be neglected. We will thus ignore the spin dependence of $y_{0}(k)$ in the following, as well as the term quadratic in $\alpha_{v}$ and $\alpha_{l}$ in Eq. (11), since it has the same order of magnitude. In the basis $\{|m, n, k, \sigma\rangle\}(\sigma=\uparrow, \downarrow)$, the full Hamiltonian (9) has thus the matrix elements,

$$
\begin{aligned}
& \langle m, n, k|H| m, n, k\rangle \\
& \simeq\left(\begin{array}{cc}
E_{m n k}-\frac{g \mu_{B} B}{2}+\alpha_{l} \frac{\omega_{y}^{2}}{\Omega^{2}} k & -i \alpha_{v} \frac{\omega_{y}^{2}}{\Omega^{2}} k \\
i \alpha_{v} \frac{\omega_{y}^{2}}{\Omega^{2}} k & E_{m n k}+\frac{g \mu_{B} B}{2}-\alpha_{l} \frac{\omega_{y}^{2}}{\Omega^{2}} k
\end{array}\right),
\end{aligned}
$$

where

$$
E_{m n k}=\frac{\hbar^{2} k^{2}}{2 m_{x}} \frac{\omega_{y}^{2}}{\Omega^{2}}+\hbar \Omega\left(m+\frac{1}{2}\right)+\hbar \omega_{z}\left(2 n+\frac{3}{2}\right)-\mu
$$

are the energies of the states $|m, n, k\rangle$. In the absence of SOC (i.e., for $\alpha_{v}=\alpha_{l}=0$ ), the states $|m, n, k, \sigma\rangle$ are the eigenstates of $H$ with energies,

$$
\xi_{m n \sigma k}=E_{m n k}-s(\sigma) g \mu_{B} B,
$$

where $s(\downarrow)=-1 / 2$ and $s(\uparrow)=1 / 2$. The presence of an applied lateral or vertical electric field, respectively, alters the Zeeman splitting energy or mixes the different spin species within a given transverse mode $|m, n\rangle$.

The transport properties of the waveguide can be computed directly from the model above. Of particular interest is the conductance $G(\mu)=d I(\mu) / d V$, corresponding to the derivative of the current in the waveguide (which depends on its chemical potential $\mu$ ) with respect to the bias voltage $V$. We focus here on the conductance at zero bias $(V=0)$. Increasing the chemical potential increases the conductance by one quantum $e^{2} / h$ each time a new eigenstate of $H$ is populated, as can be obtained via Landauer theory [45]. In practice, we calculate the eigenvalues of $H$ as a function of $k$ and count the number of times they cross the zero-energy axis, indicating the position of the Fermi momenta. Figure 3 shows the conductance as a function of the out-of-plane magnetic field $B$ and chemical potential $\mu$ of a waveguide without SOC $\left[\alpha_{v}=\alpha_{l}=0\right.$, panel (a)], with SOC along $\sigma_{y}\left[\alpha_{v} \neq 0\right.$ and $\alpha_{l}=0$, panel (b)] and with SOC along $\sigma_{z}\left[\alpha_{v}=0\right.$ and $\alpha_{l} \neq 0$, panel (c)], for typical other parameters, which can be found, e.g., by fitting experimental data as in $[11,12,46]$. In the absence of SOC, the conductance increases monotonically as a function of $\mu$-in steps of $2 e^{2} / h$ at $B=0$ and steps of $e^{2} / h$ for $B>0$ due to lifting of the spin degeneracy by the Zeeman term-as the transverse modes $|m, n\rangle$ are gradually populated. 
(a)

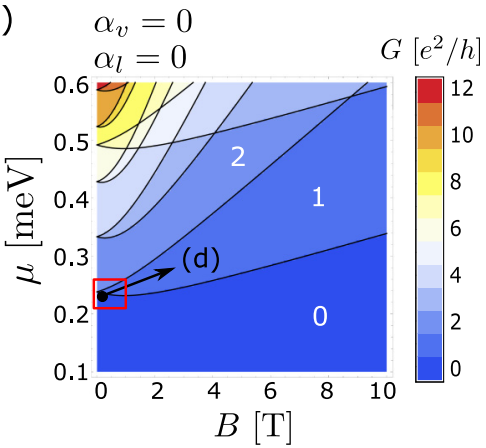

(b) $\quad \alpha_{v}=0.5 \mathrm{meV} \mathrm{nm}$

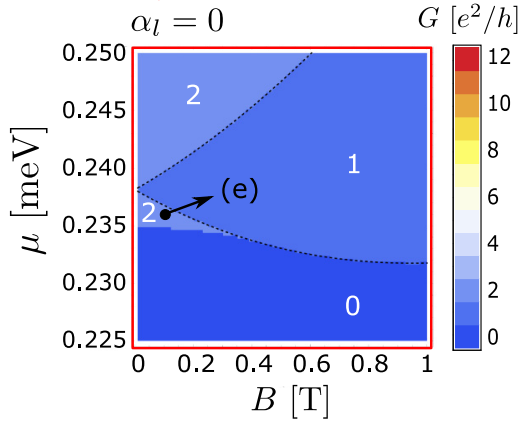

$G\left[e^{2} / h\right]$

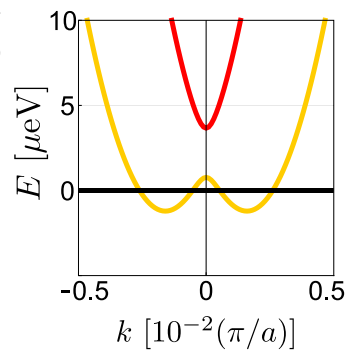

(c)

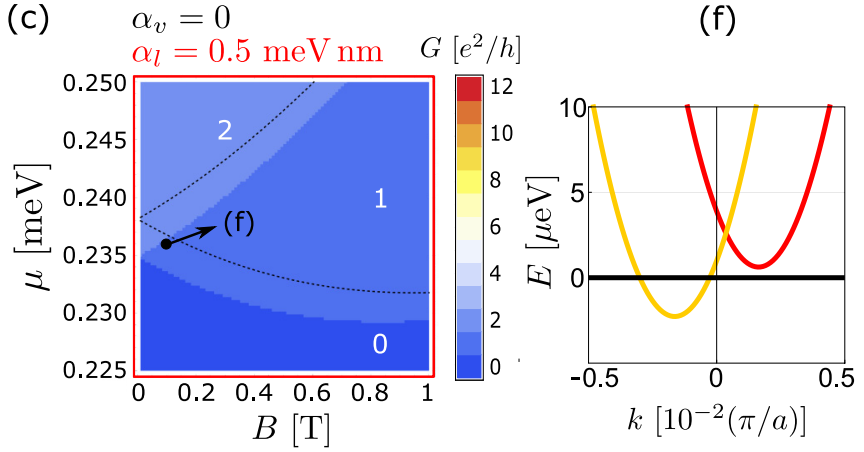

FIG. 3. Zero-bias conductance with and without SOC. Conductance $G$ (in units of $e^{2} / h$ ) as a function of magnetic field $B$ and chemical potential $\mu$ for $\alpha_{v}=\alpha_{l}=0 \mathrm{meV} \mathrm{nm} \mathrm{(a),} \alpha_{v}=0.5 \mathrm{meV} \mathrm{nm}$ and $\alpha_{l}=0 \mathrm{meV} \mathrm{nm} \mathrm{(b),} \mathrm{and} \alpha_{v}=0 \mathrm{meV} \mathrm{nm}$ and $\alpha_{l}=0.5 \mathrm{meV} \mathrm{nm}$ (c). The dashed black lines in panels (b) and (c) correspond to the borders of the conductance regions in the absence of SOC [solid lines in (a)]. Panels (d), (e), and (f) show the two dispersion relations associated with the eigenstates of $\langle 0,0, k|H| 0,0, k\rangle$ related to panels (a), (b), and (c), respectively, for $B=0.1 \mathrm{~T}$ and $\mu=0.236 \mathrm{meV}$ [black dots in panels (a)-(c)]. We chose typical waveguide parameters [11,12] $m_{x}=m_{y}=m_{z} / 3=2 m_{e}$ where $m_{e}$ is the electron mass, $l_{y}=\sqrt{\hbar /\left(m_{y} \omega_{y}\right)}=20 \mathrm{~nm}, l_{z}=\sqrt{\hbar /\left(m_{z} \omega_{z}\right)}=10 \mathrm{~nm}, g=0.5$.

This case has been studied in detail in [11,12]. Figure 3(d) shows the two dispersion relations of the electrons in the transverse mode $|0,0\rangle$ for a value $(B, \mu)$ just below the threshold necessary to populate one of the bands. The presence of SOC perturbs the dispersion relations. For $\alpha_{v} \neq 0$, a gap opens at low $B$ in the single-particle dispersion relations. This can lead to a situation where the lower one exhibits four crossing with the zero-energy axis [see Fig. 3(e)], which is known to lead to a conductance of $2 e^{2} / h$ (see, e.g., [47]), as shown in Fig. 3(b). As a consequence, the conductance increase as a function of $\mu$ is no longer monotonic. For $\alpha_{l} \neq 0$, the dispersion relations become asymmetric in $k$ and are sightly pushed downward. This effect is likely to populate one of the bands as shown in Fig. 3(f) and thus increase the conductance at lower chemical potential than one would have expected in the absence of SOC.

\section{B. Electron-electron interactions}

We now discuss how to model the effects of attractive interactions between the electrons in the waveguide, in order to study how they affect the single-particle picture presented above. For waveguides written on $\mathrm{SrTiO}_{3}$-based interfaces, the precise form and origin of the interactions is unfortunately still an open question. It is known there are gate-tunable electron-electron interactions in nanostructures created on the LAO/STO interface [9], but the question of the pairing mechanism has not been resolved. Hence, we proceed below with a simple mean-field model to draw qualitative predictions on the effects of attractive interactions on the transport properties of the waveguide, which we believe are generic for such systems and consistent with more elaborate models.

We will focus in the following on regions of parameters where only two subbands labeled as $\alpha=|m, n, \downarrow\rangle$ and $\beta=\left|m^{\prime}, n^{\prime}, \uparrow\right\rangle$ are relevant [see, e.g., $|0,0, \downarrow\rangle$ and $|0,0, \uparrow\rangle$ at $B \simeq 0$ in Fig. 3(a), or $|0,1, \downarrow\rangle$ and $|1,0, \uparrow\rangle$ around $B \simeq 2.5$ $\mathrm{T}$ and $\mu \simeq 0.5 \mathrm{meV}$ ]. As shown in Ref. [11], locking of such sub-bands was observed due to the presence of attractive interactions. Our goal here is to analyze how SOC will affect the electron pairing in these regions. We consider an interaction Hamiltonian in second quantization and at the mean-field level of the form,

$$
\begin{gathered}
H_{I}=\sum_{k}\left[\sum_{\gamma=\alpha, \beta} \Sigma_{\gamma} c_{\gamma k}^{\dagger} c_{\gamma k}-\left(\chi c_{\alpha k}^{\dagger} c_{\beta k}+\text { H.c. }\right)\right. \\
\left.+\left(\Delta c_{\alpha k}^{\dagger} c_{\beta-k}^{\dagger}+\text { H.c. }\right)\right],
\end{gathered}
$$

where $c_{k \alpha}$ is the annihilation operator of an electron in the sub-band $\alpha$ with a wave vector $k$, and where $\Sigma_{\gamma}, \chi$, and $\Delta$ are the Hartree, Fock, and Bogoliubov mean fields defined as

$$
\begin{aligned}
\Sigma_{\gamma} & =\frac{U}{2 \pi} \int_{-\infty}^{\infty}\left\langle c_{\bar{\gamma} k}^{\dagger} c_{\bar{\gamma} k}\right\rangle d k, \\
\chi & =\frac{U}{2 \pi} \int_{-\infty}^{\infty}\left\langle c_{\alpha k}^{\dagger} c_{\beta k}\right\rangle d k, \\
\Delta & =\frac{U}{2 \pi} \int_{-\infty}^{\infty}\left\langle c_{\beta k} c_{\alpha-k}\right\rangle d k,
\end{aligned}
$$

where $\bar{\gamma}$ denotes the opposite sub-band of $\gamma(\gamma=\alpha, \beta)$ and where $U$ is the interaction strength.. The mean fields have to be found self-consistently as explained in the next subsection. The interaction Hamiltonian [Eq. (18)] can be viewed as originating from the interaction term of a twoband, one-dimensional Hubbard model [11], or from a contact interaction, which is the usual form of interactions in dilute cold atomic gases. In Appendix B, we present a derivation of Eq. (18) from such an assumption, relevant for these systems. In our model, we consider that $U$ has the following empirical 
scaling with the magnetic field:

$$
U \equiv U(B)=U_{0} \sqrt{1-\frac{\omega_{c}^{2}}{\Omega^{2}}}=U_{0} \frac{\omega_{y}}{\Omega},
$$

where $U_{0} \equiv U(0)$ is a bare interaction strength (in dimensions of energy $\times$ length). This makes $|U|$ decreasing as a function of the magnetic field. A physical justification is the following: The effective kinetic energy of the electrons of our model [see Eq. (16)] scales with the magnetic field as $\omega_{y}^{2} / \Omega^{2}$, so that electron momenta behave as $k \sim \sqrt{2 m_{x} E / \hbar^{2}}\left(\Omega / \omega_{y}\right)$. The scaling of $U$ is such that it compensates the increase with magnetic field of the typical range of momenta involved in the interactions. This has the effect of keeping the mean fields (19)-(21) $\Delta, \Sigma, \chi \propto U \int \cdot d k$ independent of this effective scaling (it amounts to a contraction of the real-space quantization length $L$ reciprocally associated with $k$ ). Our phenomenological scaling has allowed us to obtain results in qualitative agreement with experimental data [23]. While other scalings with the magnetic field could be chosen and possibly compatible with experimental data, a parameter $U$ without scaling with $B$ at all would lead to the unphysical situation where the resulting pairing of electrons $\Delta$ would increase with $B$ in an unbounded way. We also checked that the findings presented below remain qualitatively the same for other choices of scaling. Finally, in the case of SOC produced via modulations, it would be reasonable to consider a modulated interaction as in [48], where it has been shown that such assumption can produce fractional conductance plateaux, compatible with the experimental data presented in $[22,23]$.

Note that our model for the interactions is not a BCS model for superconductivity $[49,50]$, but instead a model to describe electron pairing without superconductivity. Indeed, in one dimension, phase fluctuations are expected to preclude the establishment of a long-range order required for superconductivity [51]. However, there is evidence that electron pairs can survive in its absence, notably in LAO/STO interfaces [46,52]. This is what our model aims to describe qualitatively. Hence, here, a nonzero value of $\Delta$ should not be interpreted as a signature of superconductivity, but rather as a signature of electron pairing.

\section{Hartree-Fock-Bogoliubov model}

We now add to Eq. (18) the single-particle Hamiltonian (9) for sub-bands $\alpha=|m, n, \downarrow\rangle$ and $\beta=\left|m^{\prime}, n^{\prime}, \uparrow\right\rangle$ only, which in second quantization reads

$$
\begin{aligned}
H= & \int d x\left\{\Psi^{\dagger}(x)\left[\left(-\frac{\hbar^{2} \nabla^{2}}{2 m_{x}} \frac{\omega_{y}^{2}}{\Omega^{2}}-\mu\right) \mathbb{1}_{2}+\left(\alpha_{v} \frac{\omega_{y}^{2}}{\Omega^{2}} \frac{\nabla}{i}\right) \sigma_{y}+\left(-\frac{g \mu_{B} B}{2}+\alpha_{l} \frac{\omega_{y}^{2}}{\Omega^{2}} \frac{\nabla}{i}\right) \sigma_{z}\right] \Psi(x)\right. \\
& \left.+\left[\hbar \Omega\left(m+\frac{1}{2}\right)+\hbar \omega_{z}\left(2 n+\frac{3}{2}\right)\right] \Psi_{\alpha}^{\dagger}(x) \Psi_{\alpha}(x)+\left[\hbar \Omega\left(m^{\prime}+\frac{1}{2}\right)+\hbar \omega_{z}\left(2 n^{\prime}+\frac{3}{2}\right)\right] \Psi_{\beta}^{\dagger}(x) \Psi_{\beta}(x)\right\},
\end{aligned}
$$

where $\Psi(x)=\left(\Psi_{\beta}(x), \Psi_{\alpha}(x)\right)^{T}$ is the vector of 1D field operators $\Psi_{\gamma}(x)$ for electrons in sub-bands $\gamma$. Using the Fourier decomposition,

$$
\Psi_{\gamma}(x)=\frac{1}{\sqrt{L}} \sum_{k} c_{\gamma k} e^{i k x}
$$

where $L$ is the quantization length, Eq. (23) together with the interaction Hamiltonian (18) yields the Hartree-FockBogoliubov model,

$$
H_{\mathrm{HFB}}=H+H_{I}=\sum_{k}\left[\sum_{\gamma=\alpha, \beta}\left(\xi_{\gamma k}+\Sigma_{\gamma}+2 s(\gamma) \frac{\omega_{y}^{2}}{\Omega^{2}} \alpha_{l} k\right) c_{\gamma k}^{\dagger} c_{\gamma k}+\left[\left(i \alpha_{v} \frac{\omega_{y}^{2}}{\Omega^{2}} k-\chi\right) c_{\alpha k}^{\dagger} c_{\beta k}+\text { H.c. }\right]+\left(\Delta c_{\alpha k}^{\dagger} c_{\beta-k}^{\dagger}+\text { H.c. }\right)\right] \text {. }
$$

As can be seen in Eq. (25), the Hartree shifts and the SOC coming from a lateral electric field alter the single-particle energies $\xi_{\alpha k}$. More specifically, the Hartree shifts $\Sigma_{\alpha}$ push down the energy of the electron in the sub-band $\alpha$ due to the presence of the other electron in the sub-band $\beta$. Regarding the Fock fields, they alter the same spin-flip terms as the SOC coming from a vertical electric field.

The Hamiltonian $H_{\mathrm{HFB}}$ defines the following self-consistent eigenvalue problem in the electron and hole basis $\left\{c_{\alpha k}, c_{\alpha-k}^{\dagger}, c_{\beta k}, c_{\beta-k}^{\dagger}\right\}$,

$$
\left(\begin{array}{cccc}
\xi_{\alpha k}+\Sigma_{\alpha}-\alpha_{l} \frac{\omega_{y}^{2}}{\Omega^{2}} k & 0 & i \alpha_{v} \frac{\omega_{y}^{2}}{\Omega^{2}} k-\chi & \Delta \\
0 & -\xi_{\alpha k}-\Sigma_{\alpha}-\alpha_{l} \frac{\omega_{y}^{2}}{\Omega^{2}} k & -\Delta^{*} & -i \alpha_{v} \frac{\omega_{y}^{2}}{\Omega^{2}} k+\chi^{*} \\
-i \alpha_{v} \frac{\omega_{y}^{2}}{\Omega^{2}} k-\chi^{*} & -\Delta & \xi_{\beta k}+\Sigma_{\beta}+\alpha_{l} \frac{\omega_{y}^{2}}{\Omega^{2}} k & 0 \\
\Delta^{*} & i \alpha_{v} \frac{\omega_{y}^{2}}{\Omega^{2}} k+\chi & 0 & -\xi_{\beta k}-\Sigma_{\beta}+\alpha_{l} \frac{\omega_{y}^{2}}{\Omega^{2}} k
\end{array}\right) \phi_{j k}=E_{j k} \phi_{j k},
$$

where $E_{j k}$ and $\phi_{j k}(j=1,2)$ are the quasienergies and the quasiparticle wave functions (note that there are also two other solutions of the eigenvalue problem corresponding to the associated quasiholes). Equation (26) is the direct 

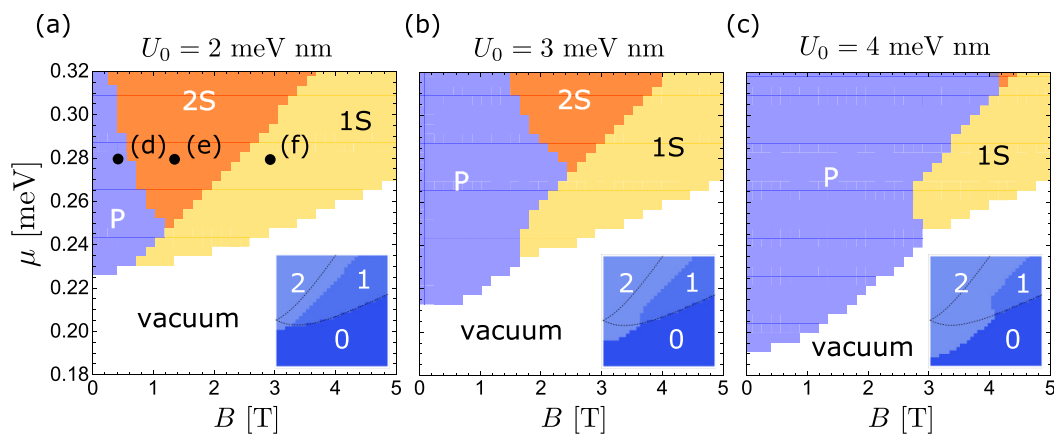

(d)

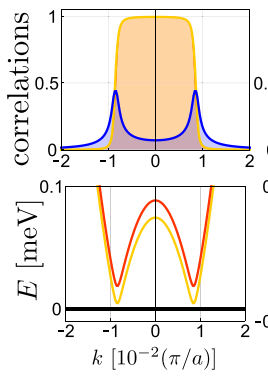

(e)

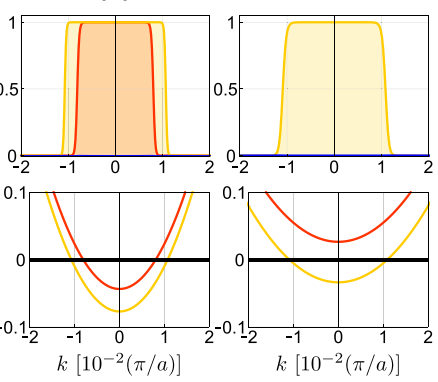

FIG. 4. Phase diagrams and zero-bias conductance with interactions and without SOC. (a)-(c) Phase diagrams of the waveguide near the crossing of the sub-bands $\alpha=|0,0, \downarrow\rangle$ and $\beta=|0,0, \uparrow\rangle$ as a function of magnetic field $B$ and chemical potential $\mu$ for different attractive interaction strength $\left|U_{0}\right|=2 \mathrm{meV} \mathrm{nm}$ (a), $3 \mathrm{meV} \mathrm{nm}$ (b), and $4 \mathrm{meV} \mathrm{nm} \mathrm{(c),} \mathrm{and} \mathrm{temperature} T=25 \mathrm{mK}$. The phase "P" corresponds to a region where $|\Delta|>10^{-3} \mathrm{meV}\left(\sim k_{B} T\right)$, i.e., when we have electron pairs. The phases " $1 \mathrm{~S}$ " and " $2 \mathrm{~S}$ " denote the phases where one- and two single-particle bands are populated, respectively. The phase "vacuum" denotes the phase where none of the single-particle bands are populated and no pairing is observed $\left(|\Delta|<10^{-3} \mathrm{meV}\right)$. The insets show the associated conductance (in units of $e^{2} / h$ ), where the thin dashed lines correspond to the noninteracting case. Panels (d)-(f) show on the top the correlations $\left\langle c_{\beta k}^{\dagger} c_{\beta k}\right\rangle$ (yellow), $\left\langle c_{\alpha k}^{\dagger} c_{\alpha k}\right\rangle$ (red), and $\left|\left\langle c_{\beta k} c_{\alpha-k}\right\rangle\right|$ (blue) appearing in the definitions of the mean fields (19)-(21), for $\mu=0.28 \mathrm{meV}$ and $B=0.5 \mathrm{~T}, 1.5 \mathrm{~T}$ and $3 T$ respectively [black dots in panel (a)]. The corresponding band structures appear at the bottom, showing, e.g., in panel (d) (bottom) that a gap $|\Delta|$ protects the band from single-particle excitations. Other parameters are as in Fig. 3.

generalization of Eq. (5) of [11] to include SOC. In order to solve Eq. (26), we need to proceed self-consistently, by starting with an initial guess of the values of the mean fields $\Sigma_{\alpha}, \chi$, and $\Delta$. These values are inserted into Eq. (26), which is then solved to obtain the quasienergies and quasiparticle wave functions $E_{j k}$ and $\phi_{j k}$ and compute new values for the mean fields using Eqs. (19)-(21). This last step is done via Bogoliubov transformation of the electron annihilation operators $c_{\alpha k}$ and $c_{\beta k}$ into quasiparticle annihilation operators $\gamma_{1 k}$ and $\gamma_{2 k}$, where we assume here the thermal state correlation functions,

$$
\begin{aligned}
& \left\langle\gamma_{i k}^{\dagger} \gamma_{j k}\right\rangle=\delta_{i j} n\left(E_{i k}\right), \\
& \left\langle\gamma_{i k} \gamma_{j k}^{\dagger}\right\rangle=\delta_{i j}\left[1-n\left(E_{i k}\right)\right], \\
& \left\langle\gamma_{i k} \gamma_{j k}\right\rangle=\left\langle\gamma_{i k}^{\dagger} \gamma_{j k}^{\dagger}\right\rangle=0,
\end{aligned}
$$

where $n(E)=1 /\left[1+e^{E /\left(k_{B} T\right)}\right]$ is the Fermi distribution with $k_{B}$ the Boltzmann constant and $T$ the temperature. The procedure is then repeated until the mean fields have converged. Note that since we work in the electron-hole basis (twice as big as the physical basis), the computed quasienergies always appear in conjugate pairs $\left(E_{1 k},-E_{1-k}\right)$ and $\left(E_{2 k},-E_{2-k}\right)$, and one has to select only one member of each pair.

\section{RESULTS}

\section{A. Without SOC}

We first show the effects of interactions in the absence of spin-orbit coupling $\left(\alpha_{v}=\alpha_{l}=0\right)$. We focus here on the two lowest sub-bands $\alpha=|0,0, \downarrow\rangle$ and $\beta=|0,0, \uparrow\rangle$ that are close from each other at low magnetic field. We solve the eigenvalue problem (26) as a function of $B$ and $\mu$ for different interaction strength $U_{0}$ until the single-particle spectra and the mean fields have converged, which allows us to construct phase diagrams and associated conductance maps, as shown in Fig. 4. As for Fig. 3, the available single-particle phases are identified from the number of crossing points with the zero-energy axis (i.e., the number of finite Fermi momenta) of the converged single-particle spectra, which indicates the presence or not of electrons in the single-particle bands. A conductance of $e^{2} / h$ is associated with each occupied band. By contrast, a pair phase is identified when a nonzero value of $\Delta$ emerges from the calculations, which corresponds to the situation where the single-particle spectra are gapped and denotes the presence of electron pairs (not superconductivity) in the waveguide. Following the Maslov and Stone theorem [53], we associate with this pair phase a conductance of $2 e^{2} / h$, as we consider that the electron pairs unbind when they reach the leads before dissipating energy [11]. The conductance of the electron pairs is thus the sum of the conductances of the individual electrons. Figures 4(a)-4(c) shows the obtained phase diagrams and conductance maps (insets) for different $U_{0}$. The insets show the associated conductance, where the conductance associated with a pair is indistinguishable from the conductance associated with the two unpaired electrons in different sub-bands. Due to the presence of the interactions, the electrons in the two first sub-bands are paired at low $B$, leading to a direct increase of $2 e^{2} / h$. For a certain value of $B$ defined as the pairing field $B_{p}$, the electron pairs are finally split, leaving us with signatures of single-electron sub-bands. Increasing $\left|U_{0}\right|$ enhanced the pairing area and thus shifts the position where the conductance line splits. In our model, the resulting pairing energy behaves as $\Delta \propto U_{0}^{2} m_{x}$, in agreement with the expected behavior $\Delta \propto U_{0}^{2} / t$ of a tight-binding model with hopping parameter $t=\hbar^{2} /\left(m_{x} a^{2}\right)$ and lattice spacing $a$ [12]. The pairs are broken when the Zeeman splitting energy $g \mu_{B} B$ compensates the pairing energy $\Delta$, which provides the typical scaling of the pairing field $B_{p} \propto U_{0}^{2} m_{x} /\left(g \mu_{B}\right)$ at which the lines split.

Figures 4(d)-4(f) shows the values of the correlations functions $\left\langle c_{\beta k}^{\dagger} c_{\beta k}\right\rangle,\left\langle c_{\alpha k}^{\dagger} c_{\alpha k}\right\rangle$, and $\left\langle c_{\beta k} c_{\alpha-k}\right\rangle$ appearing in the mean fields $\Sigma_{\alpha}, \Sigma_{\beta}$, and $\Delta$ for three pairs of values $(B, \mu)$ corresponding to three different points of the phase diagram in 
(a)

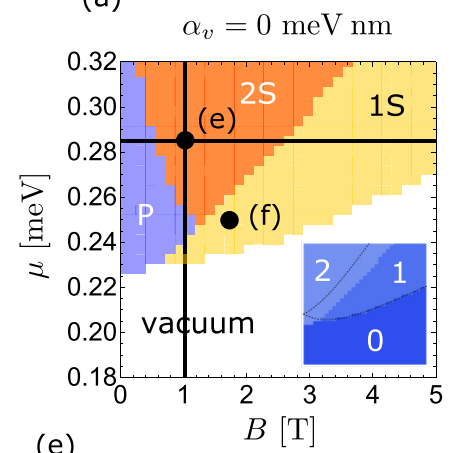

(e)
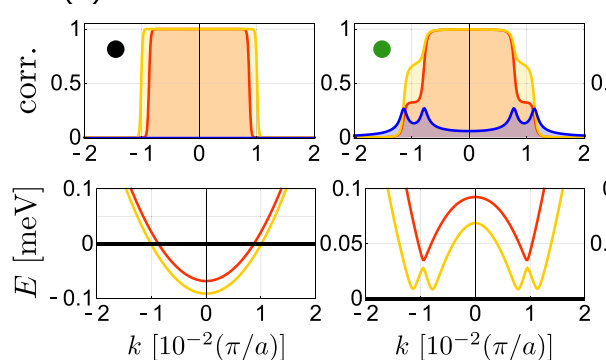

(b)

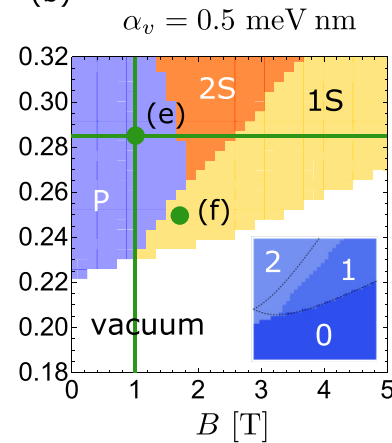

(c)

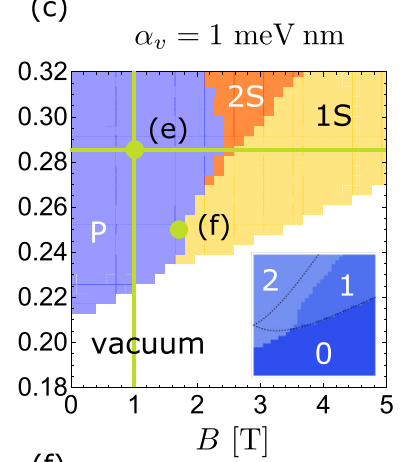

(d)
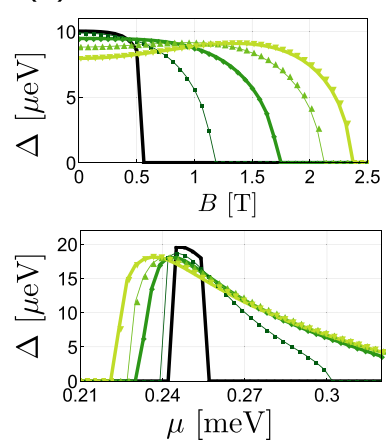

FIG. 5. Phase diagrams and zero-bias conductance with interactions and vertically induced SOC $\left(\alpha_{v} \neq 0\right)$. (a)-(c) Phase diagrams of the waveguide near the crossing of the sub-bands $\alpha=|0,0, \downarrow\rangle$ and $\beta=|0,0, \uparrow\rangle$ as a function of magnetic field $B$ and chemical potential $\mu$ for different SOC strength $\alpha_{v}=0 \mathrm{meV} \mathrm{nm}$ (a), $0.5 \mathrm{meV} \mathrm{nm}$ (b), and $1 \mathrm{meV} \mathrm{nm}$ (c), for interaction strength $\left|U_{0}\right|=2 \mathrm{meV} \mathrm{nm}$ and temperature $T=$ $25 \mathrm{mK}$. The phases are labeled as in Fig. 4, and the insets show the associated conductance. (d) Pairing $\Delta$ across horizontal (top) and vertical (bottom) line cuts of the phase diagrams for SOC strength $\left(\alpha_{v}=0,0.25,0.5,0.75\right.$, and $1 \mathrm{meV} \mathrm{nm}$ ). (e) and (f) (top) Correlations $\left\langle c_{\beta k}^{\dagger} c_{\beta k}\right\rangle$ (yellow), $\left\langle c_{\alpha k}^{\dagger} c_{\alpha k}\right\rangle$ (red), and $\left|\left\langle c_{\beta k} c_{\alpha-k}\right\rangle\right|$ (blue) appearing in the definitions of the mean fields (19)-(21), for $B=1 \mathrm{~T}$ and $\mu=0.285 \mathrm{meV}$ [panel (e)] and $B=1.75 \mathrm{~T}$ and $\mu=0.25 \mathrm{meV}$ [panel (f)], corresponding to the dots shown in panels (a-c). (e) and (f) (bottom) Corresponding band structures. Other paramaters are as in Fig. 3.

Fig. 4(a) along the horizontal line corresponding to $\mu=0.28$ $\mathrm{meV}$, one with pairing $(B=0.5 \mathrm{~T})$, one without pairing but the two sub-bands populated $(B=1.5 \mathrm{~T})$, and one without pairing and only one sub-band populated $(B=3 \mathrm{~T})$. In the absence of pairing, the correlation functions appearing in the Hartree shifts correspond to standard single-particle Fermi distributions and the one appearing in the definition of the pairing vanishes [see Figs. 4(e) and 4(f)]. In the presence of pairing, the single-particle distributions smoothen around the Fermi level, where nonvanishing pairing correlations appear [see Fig. 4(d)]. The associated single-particle spectra plotted at the bottom of the Figs. 4(d)-4(f) show clearly when electrons occupy one of the single-particle bands (f), the two single-particle bands (e), or when they are paired, as translated by the apparition of a gap that protects the bands from singleparticle excitations (d).

Note that the transport properties of the waveguide are strongly dependent on the temperature. Typical experiments in LAO/STO devices work with temperature of a few dozen of milli-Kelvin, and we chose $T=25 \mathrm{mK}$ throughout our work. Increasing the temperature destroys electron pairs typically when the thermal fluctuations overcome the pairing energy, i.e., when $k_{B} T \gtrsim|\Delta|$.

\section{B. With SOC}

\section{Vertically induced $\operatorname{SOC}\left(\alpha_{v} \neq 0\right)$}

We now study the interplay between SOC and interactions in such a waveguide model. We start with the case of a SOC engineered through an applied vertical electric field $\left(\alpha_{v} \neq 0\right)$. Figures $5(\mathrm{a})-5$ (c) show phase diagrams and associated conductance maps (in units of $e^{2} / h$ ) for different values of $\alpha_{v}$ and for $\left|U_{0}\right|=2 \mathrm{meVnm}$. As can be seen in the figures, an enhanced pairing area is obtained for increasing $\alpha_{v}$, showing that the SOC can assist pairing. This is clearly seen in Fig. 5(d) showing $\Delta$ across horizontal (top) and vertical (bottom) line cuts of the previous panels. Intuitively, this can be understood since the SOC term is proportional to $\sigma_{y}$, it provides a direct coupling between up and down electrons. In order to understand this in more detail, we plot in Figs. 5(e) and 5(f) the correlation functions $\left\langle c_{\beta k}^{\dagger} c_{\beta k}\right\rangle,\left\langle c_{\alpha k}^{\dagger} c_{\alpha k}\right\rangle$, and $\left\langle c_{\beta k} c_{\alpha-k}\right\rangle$ appearing in the definitions of the mean field $\Sigma_{\alpha}, \Sigma_{\beta}$, and $\Delta$ for two pairs of values $(B, \mu)$ corresponding to two specific points in the phase diagrams: $(B=1 \mathrm{~T}, \mu=0.285 \mathrm{meV})$ [Fig. $5(\mathrm{e})]$ and $(B=$ $1.75 \mathrm{~T}, \mu=0.25 \mathrm{meV}$ ) [Fig. 5(f)]. The different panels in Figs. 5(e) and 5(f) show, respectively, the transition occurring from the phases " $2 S$ " to "P" (e) and " $1 S$ " to "P" (f) when increasing the SOC strength. More specifically, we see that the single-particle sub-band is linearly depleted in favor of the second single-particle sub-band, even if the latter is nonoccupied without SOC. The physical picture is thus that the SOC is able to transfer electrons of one sub-band to the other one [see, e.g., middle plot in (f)], which are then likely to pair due to the interactions [see, e.g., right plot in (f)]. As mentioned in the introduction, this phenomenon is a possible interpretation of the experimental data presented in [23], showing that vertical modulations of 
(a)

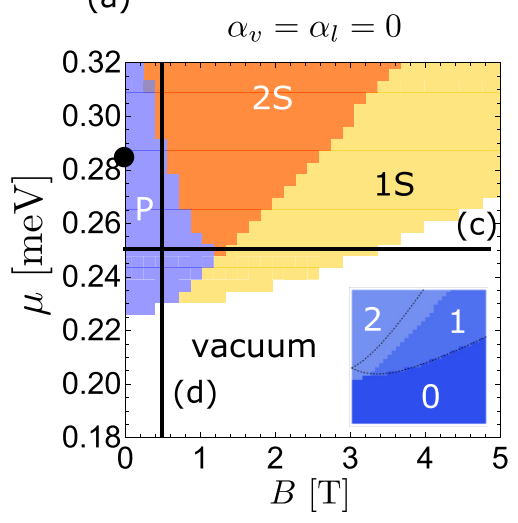

(b)

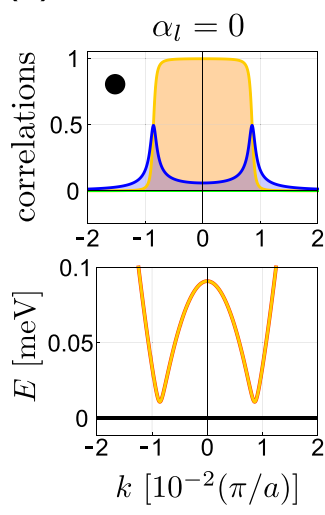

(c)
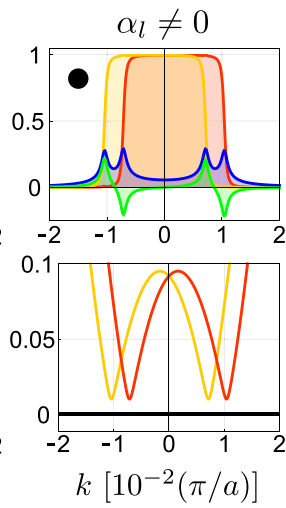

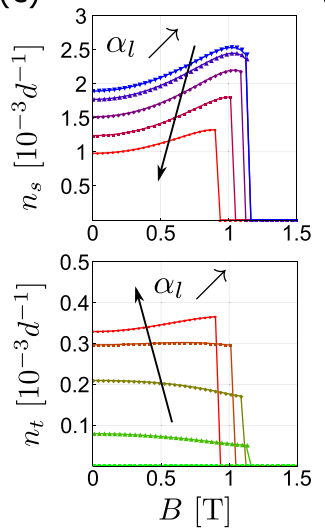

(d)
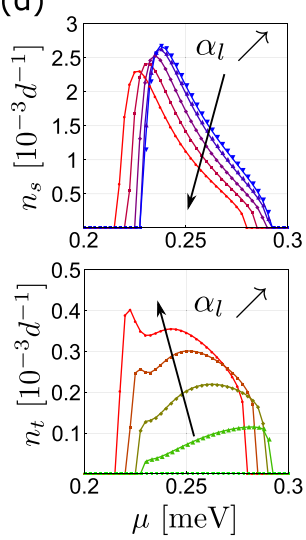

FIG. 6. Emergence of spin-triplet electron pairs via laterally induced SOC $\left(\alpha_{l} \neq 0\right)$. (a) Phase diagram of the waveguide near the crossing of the sub-bands $\alpha=|0,0, \downarrow\rangle$ and $\beta=|0,0, \uparrow\rangle$ as a function of magnetic field $B$ and chemical potential $\mu$ for SOC strength $\alpha_{v}=\alpha_{l}=$ $0 \mathrm{meV} \mathrm{nm}$, interaction strength $\left|U_{0}\right|=2 \mathrm{meV} \mathrm{nm}$, and temperature $T=25 \mathrm{mK}$ [same as Figs. 4(a) and 5(a)]. (b) Correlations $\left\langle c_{\beta k}^{\dagger} c_{\beta k}\right\rangle$ (yellow), $\left\langle c_{\alpha k}^{\dagger} c_{\alpha k}\right\rangle$ (red), $\left|\left\langle c_{\beta k} c_{\alpha-k}-c_{\alpha k} c_{\beta-k}\right\rangle / 2\right|$ (blue), and $\left\langle c_{\beta k} c_{\alpha-k}+c_{\alpha k} c_{\beta-k}\right\rangle / 2$ (green) as a function of $k$ for $B=0$ and $\mu=0.285$ meV nm [black dot in (a)] and SOC strength $\alpha_{l}=0$ (left) and $\alpha_{l}=0.5 \mathrm{meV} \mathrm{nm}$ (right). Note that the global phase of the initial mean-field values of the self-consistent problem has been chosen so that $\left\langle c_{\beta k} c_{\alpha-k}+c_{\alpha k} c_{\beta-k}\right\rangle / 2$ is real. The SOC induces the generation of spin-triplet electron pairs. (c) Density of singlet (top) and triplet (bottom) as a function of $B$ for $\mu=0.25 \mathrm{meV} \mathrm{nm}$ along the horizontal black line of (a) for $\alpha_{l}=0$, 0.25 , 0.5, 0.75, $1 \mathrm{meV} \mathrm{nm}$ [from blue to red (top) and from green to red (bottom)]. (d) Density of spin-singlet (top) and spin-triplet (bottom) as a function of $\mu$ for $B=0.5 \mathrm{~T}$ along the vertical black line of (a) for $\alpha_{l}=0,0.25,0.5,0.75,1 \mathrm{meV}$ nm [from blue to red (top) and from green to red (bottom)]. Other parameters are as in Fig. 3.

the c-AFM tip producing the electron waveguide lead to an enhanced pairing of the two lowest sub-bands up to very large magnetic field (as large as $B=16 \mathrm{~T}$ in some devices) compared to unmodulated devices. More broadly, our findings are consistent with other studies, as enhanced pairing due to SOC has been found in other contexts, such as in superconductors with magnetic impurities [54] and in cold atoms [34,35].

\section{Laterally induced $\operatorname{SOC}\left(\alpha_{l} \neq 0\right)$}

We now investigate the case of SOC coming from an applied electric field along $y\left(\alpha_{l} \neq 0\right)$. In contrast to a vertically induced SOC, a laterally induced SOC does not lead to an increase of the pairing area "P." However, it allows for the emergence of triplet pairs of electrons in the area where the usual pairing defined through nonzero values of $\Delta$ is usually observed, which is a singlet pairing by construction. Indeed, using the fermionic nature of the electron annihilation operators, we can rewrite without restriction,

$$
\begin{aligned}
\Delta & =\frac{U}{2 \pi} \int\left\langle c_{\beta k} c_{\alpha-k}\right\rangle d k \\
& =\frac{U}{4 \pi} \int\left[\left\langle c_{\beta k} c_{\alpha-k}\right\rangle-\left\langle c_{\alpha-k} c_{\beta k}\right\rangle\right] d k, \\
& =\frac{U}{4 \pi} \int\left[\left\langle c_{\beta k} c_{\alpha-k}\right\rangle-\left\langle c_{\alpha k} c_{\beta-k}\right\rangle\right] d k,
\end{aligned}
$$

showing that $\Delta$ comes from the expectation value of the spinsinglet pair operator,

$$
s_{k}=\frac{c_{\beta k} c_{\alpha-k}-c_{\alpha k} c_{\beta-k}}{\sqrt{2}} .
$$

Due to the presence of a laterally induced SOC, it is legitimate (see, e.g., $[34,35]$ ) to also look at the presence of spin triplet pairs defined through the spin-triplet operator,

$$
t_{k}=\frac{c_{\beta k} c_{\alpha-k}+c_{\alpha k} c_{\beta-k}}{\sqrt{2}}
$$

In the absence of a laterally induced SOC, the single-particle spectra are symmetric under the transformation $k \rightarrow-k$ [see, e.g., Figs. 3(d) and 3(e)] and we thus have $\left\langle c_{\beta k} c_{\alpha-k}\right\rangle=$ $\left\langle c_{\beta-k} c_{\alpha k}\right\rangle=-\left\langle c_{\alpha k} c_{\beta-k}\right\rangle$ so that $\left\langle t_{k}\right\rangle=0 \forall k$. In other words, there are no spin-triplet pairs. However, for $\alpha_{l} \neq 0$, the single-particle spectra become asymmetric [see e.g., Fig. 3(f)] so that $\left\langle c_{\beta k} c_{\alpha-k}\right\rangle \neq\left\langle c_{\beta-k} c_{\alpha k}\right\rangle$ and nonzero values of $\left\langle t_{k}\right\rangle$ are likely to emerge, signaling the presence of triplet pairs in the waveguide. This is what we observed in Fig. 6. Figure 6(b) shows the correlations $\left\langle c_{\beta k}^{\dagger} c_{\beta k}\right\rangle,\left\langle c_{\alpha k}^{\dagger} c_{\alpha k}\right\rangle,\left\langle c_{\beta k} c_{\alpha-k}-\right.$ $\left.c_{\alpha k} c_{\beta-k}\right\rangle / 2$, and $\left\langle c_{\beta k} c_{\alpha-k}+c_{\alpha k} c_{\beta-k}\right\rangle / 2$ as a function of $k$ without SOC (left) and with SOC (right), for $\left|U_{0}\right|=2 \mathrm{meVnm}$ and $B=0$. Note that the phase diagram in (a) is the same as in Figs. 4(a) and 5(a) and is there for comparison. The correlations in (b) are evaluated at the coordinates $B$ and $\mu$ of the black dot in (a). The SOC splits the Fermi distributions of the single particles and the triplet pair (green) that appears is an odd function of $k$, by contrast with the singlet pair (blue) which is even, as expected (see, e.g., [55]). As a consequence, any pairing term of a form $\propto \int d k\left\langle t_{k}\right\rangle$ (similar to the singlet pairing $\Delta$ ) vanishes. We compare here the densities of singlet and triplet pairs defined as

$$
\begin{aligned}
& n_{s}=\frac{1}{2 \pi} \int\left|\left\langle s_{k}\right\rangle\right|^{2} d k, \\
& n_{t}=\frac{1}{2 \pi} \int\left|\left\langle t_{k}\right\rangle\right|^{2} d k,
\end{aligned}
$$


in Fig. 6 as a function of $B$ for $\mu=0.25 \mathrm{meV} \mathrm{nm} \mathrm{(c)} \mathrm{and}$ as a function of $\mu$ for $B=0.5 \mathrm{~T}$ (d), for $\left|U_{0}\right|=2 \mathrm{meV} \mathrm{nm}$, and different SOC strength $\alpha_{l}$. The SOC can generate a nonnegligible fraction of triplet pairs compared to singlet pairs. Also, it shifts the pairing area slightly downward along $\mu$, and contracts it as a function of $B$. Hence, it does not increase the critical magnetic fields defining the pairing area. This is consistent with experimental observations in laterally modulated waveguides that are believed to generate such a SOC [22], since such devices do not exhibit enhanced pairing fields compared to unmodulated waveguide. Our model suggests, however, that triplet pairing could potentially be observed at low magnetic field and signatures of this could thus be investigated in future experiments. Note that the emergence of triplet pairing due to SOC discussed here is also consistent with previous studies in other two- and three-dimensional condensed-matter systems [56-65].

\section{CONCLUSION}

We showed that the confinement of the electrons in 1D channels (as is found in waveguides produced, e.g., at an LAO/STO interface) is likely to reduce the intrinsic Rashba SOC felt by the electrons in the lowest band. Then, after discussing ways to recover and engineer different forms of SOC, we showed that spin-singlet and spin-triplet electron pairs can be controlled via SOC mechanisms. In particular, we showed that vertically induced SOC, which are of the Rashba form, can stabilize spin-singlet electron pairs over a larger range of applied magnetic field and gate-tunable chemical potential. By contrast, laterally induced SOC does not increase the parameter space where spin-singlet electron pairs are stable-it even contracts it-but rather, generates spin-triplet electron pairs. Our model is based on a self-consistent Hartree-FockBogoliubov that we generalized to include SOC, and our results are consistent with recent experiments [22,23] that are believed to engineer the forms of SOCs investigated in this work.

Our work provides a qualitative framework to study pairing mechanisms in 1D waveguides with attractive interactions and SOC. It would be interesting in the future to explore different models for the interactions or the coupling between different transverse modes that appear for larger SOC strength. Also, it would be interesting to use a Luttinger liquid formalism to study the effects of fluctuations beyond mean field and explore the impact of stronger electron-electron interactions [1].

Our results showed that the collective spin of electron pairs can be controlled via SOC. Knowing that bound states of more than two electrons can be realized in LAO/STO devices [12], it would be worthwhile to explore how SOC could produce and engineer exotic collective spin states of higher spin quantum numbers. In addition, with the simultaneous presence of SOC, interactions, and magnetic field, 1D waveguides written on the LAO/STO interface constitute interesting candidates for investigating the physics of Majorana fermions [66,67].

Finally, our findings could be explored in other 1D platforms for fermionic particles with attractive interactions, such as cold atoms. This could make it possible to explore different forms of interactions, and provide new ways to control the phases and transport properties in these systems using, e.g., dissipation engineering [68,69], measurements [70,71], and feedback [72,73]. The diagnostic of the impact of dissipation on these systems constitutes thus an interesting future project and a first step in these perspectives.

All data underpinning this publication are openly available from the University of Strathclyde KnowledgeBase at https:// doi.org/10.15129/10582f3d-9be6-4b85-978b-b2491c102adc.

\section{ACKNOWLEDGMENTS}

F.D., E.M., and A.J.D. acknowledge Johannes Kombe for helpful discussions. Work at the University of Strathclyde was supported by the EPSRC Programme Grant DesOEQ (Grant No. EP/P009565/1), and by AFOSR Grant No. FA9550-18-1-0064. J.L. acknowledges support from a Vannevar Bush Faculty Fellowship (ONR Grant No. N0001415-1-2847), and the National Science Foundation (Grant No. PHY-1913034). F.D. acknowledges the Belgian F.R.S.-FNRS for financial support.

\section{APPENDIX A}

We discuss here in which conditions the quadratic terms in $\alpha_{v}$ and $\alpha_{l}$ in Eq. (11) and the last term of (14) can be neglected.

Suppose that $\alpha_{v}=0$ and $\alpha_{l} \neq 0$. The eigenstate of the confining potential along $y$ will be of the form,

$$
\begin{aligned}
\phi_{m}^{\sigma}(y)= & \left(\frac{\Omega m_{y}}{\pi \hbar}\right)^{\frac{1}{4}} \frac{1}{2^{m} m !} e^{-\frac{m_{y} \Omega}{2 \hbar}\left(y-y_{0}^{\sigma}(k)\right)^{2}}, \\
& \times H_{m}\left(\sqrt{\frac{m_{y} \Omega}{\hbar}}\left[y-y_{0}^{\sigma}(k)\right]\right),
\end{aligned}
$$

where $H_{m}$ are the standard Hermite polynomials and where $y_{0}^{\sigma}(k)(\sigma=\downarrow, \uparrow)$ are their spin-dependent center of masses,

$$
\begin{aligned}
& y_{0}^{\uparrow}(k) \equiv\left\langle\uparrow\left|y_{0}(k)\right| \uparrow\right\rangle=\frac{\hbar \omega_{c} k}{\sqrt{m_{y} m_{x} \Omega^{2}}}+\frac{e B}{\hbar m_{y} \Omega^{2}} \alpha_{l}, \\
& y_{0}^{\downarrow}(k) \equiv\left\langle\downarrow\left|y_{0}(k)\right| \downarrow\right\rangle=\frac{\hbar \omega_{c} k}{\sqrt{m_{y} m_{x} \Omega^{2}}}-\frac{e B}{\hbar m_{y} \Omega^{2}} \alpha_{l} .
\end{aligned}
$$

We study below in which conditions the two eigenstates will have an overlap close to the identity, which will correspond to the parameter regime where their spin dependence can be neglected. For the ground state $(m=0)$, we have

$$
\int_{-\infty}^{\infty} \phi_{0}^{\uparrow}(y) \phi_{0}^{\downarrow *}(y) d y=e^{-\frac{m_{y}\left(y_{0}^{\uparrow}(k)-y_{0}^{\downarrow}(k)\right)^{2} \Omega}{4 \hbar}}=e^{-\frac{e^{2} B^{2} \alpha_{l}^{2}}{m_{y} \hbar^{3} \Omega^{3}}} .
$$

Figure 7 shows the right-hand side of Eq. (A3) as a function of the magnetic field $B$ for different values of $\alpha_{l}$ and for $m_{y}=$ $2 m_{e}$, where $m_{e}$ is the electron mass and $l_{y}=\sqrt{\hbar /\left(m_{y} \omega_{y}\right)}=$ $20 \mathrm{~nm}$. As can be seen in the figure, the overlap factor is equal to one within $10 \%$ error maximum for $\alpha_{l} \lesssim 1 \mathrm{meV} \mathrm{nm}$. It is thus reasonable to neglect the spin-dependent term of the center-of-mass positions for small spin-orbit coupling strength $\alpha_{l}$. For consistency, we also neglect the term $-e^{2} B^{2} \alpha_{l}^{2} /\left(2 m_{y} \Omega^{2} \hbar^{2}\right)$ in Eq. (11), which is small in a similar way compared to the energy $\hbar \Omega$ of the effective harmonic trapping along $y$, as we have $e^{2} B^{2} \alpha_{l}^{2} /\left(2 m_{y} \hbar^{3} \Omega^{3}\right) \ll 1$ [compare to Eq. (A3)]. The reasoning above also holds for $\alpha_{v} \neq 0$ and $\alpha_{l}=0$, as it yields the same order of magnitude. 


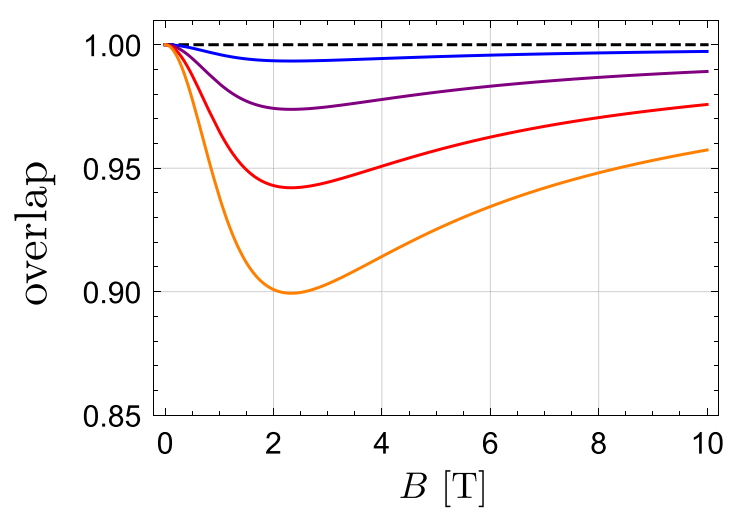

FIG. 7. Overlap [Eq. (A3)] between the two spin-dependent eigenstates of along $y$ as a function of magnetic field $B$ for $\alpha_{l}=0$ (dashed black), 0.25 (blue), 0.5 (purple), 0.75 (red), and 1 (orange) $\mathrm{meV} \mathrm{nm}$, for $m_{y}=2 m_{e}$, where $m_{e}$ is the electron mass and $l_{y}=$ $\sqrt{\hbar /\left(m_{y} \omega_{y}\right)}=20 \mathrm{~nm}$.

\section{APPENDIX B}

We derive here the interaction Hamiltonian (18) from the assumption of contact interactions, as it is usually the case in cold atoms. The starting point is the interaction Hamiltonian,

$$
H_{I}=U \int \Psi_{\alpha}^{\dagger}(x) \Psi_{\beta}^{\dagger}(x) \Psi_{\beta}(x) \Psi_{\alpha}(x) d x,
$$

where $\Psi_{\alpha}(x)$ and $\Psi_{\beta}(x)$ are the field operators for electrons in the sub-bands $\alpha$ and $\beta$, respectively.

We first perform a mean-field treatment similar to the one used in BCS theory $[49,50]$ that supposes that some operator $O$ can be written as $O=\langle O\rangle+\delta O$, i.e., quantum fluctuations $\delta O$ around an expectation value $\langle O\rangle$. By performing this treatment on pairs of operators, we obtain a quadratic form for the interactions. Explicitly, using Wick's theorem on Eq. (B1), we have

$$
\begin{aligned}
\Psi_{\alpha}^{\dagger} \Psi_{\beta}^{\dagger} \Psi_{\beta} \Psi_{\alpha} \approx & \left\langle\Psi_{\alpha}^{\dagger} \Psi_{\beta}^{\dagger}\right\rangle \Psi_{\beta} \Psi_{\alpha}+\left\langle\Psi_{\beta} \Psi_{\alpha}\right\rangle \Psi_{\alpha}^{\dagger} \Psi_{\beta}^{\dagger} \\
& -\left\langle\Psi_{\alpha}^{\dagger} \Psi_{\beta}\right\rangle \Psi_{\beta}^{\dagger} \Psi_{\alpha}-\left\langle\Psi_{\beta}^{\dagger} \Psi_{\alpha}\right\rangle \Psi_{\alpha}^{\dagger} \Psi_{\beta} \\
& +\left\langle\Psi_{\alpha}^{\dagger} \Psi_{\alpha}\right\rangle \Psi_{\beta}^{\dagger} \Psi_{\beta}+\left\langle\Psi_{\beta}^{\dagger} \Psi_{\beta}\right\rangle \Psi_{\alpha}^{\dagger} \Psi_{\alpha} .
\end{aligned}
$$

This expression contains three kinds of mean fields: (i) Hartree terms like $\left\langle\Psi_{\alpha}^{\dagger} \Psi_{\alpha}\right\rangle$, which correspond to the shift in energy due to the presence of another particle in a nearby energy band; (ii) Fock field terms like $\left\langle\Psi_{\alpha}^{\dagger} \Psi_{\beta}\right\rangle$, which correspond to spin flips; (3) Bogoliubov pairing terms $\left\langle\Psi_{\alpha}^{\dagger} \Psi_{\beta}^{\dagger}\right\rangle$, which correspond to the energy associated with forming a pair.

We then make a second approximation by considering a smoothed version of the mean fields $\langle\cdot\rangle$ appearing in Eq. (B2), obtained by averaging them over the quantization length $L$, i.e.,

$$
\langle\cdot\rangle \approx \overline{\langle\cdot\rangle}=\frac{\int\langle\cdot\rangle d x}{L},
$$

which amounts to neglect density fluctuations. Using the Fourier decomposition of the wave function $\Psi_{\gamma}(x)$ given by

$$
\Psi_{\gamma}(x)=\frac{1}{\sqrt{L}} \sum_{k} c_{\gamma k} e^{i k x},
$$

the Hamiltonian (B1) together with the approximations (B2) and (B3) can finally be rewritten as Eq. (18).
[1] T. Giamarchi, Quantum Physics in One Dimension, Vol. 121 (Oxford University Press, Oxford, 2003).

[2] A. Ohtomo and H. Y. Hwang, Nature (London) 427, 423 (2004).

[3] Y.-Y. Pai, A. Tylan-Tyler, P. Irvin, and J. Levy, Rep. Prog. Phys. 81, 036503 (2018).

[4] N. Reyren, S. Thiel, A. D. Caviglia, L. F. Kourkoutis, G. Hammerl, C. Richter, C. W. Schneider, T. Kopp, A.-S. Rüetschi, D. Jaccard, M. Gabay, D. A. Muller, J.-M. Triscone, and J. Mannhart, Science 317, 1196 (2007).

[5] S. Thiel, G. Hammerl, A. Schmehl, C. W. Schneider, and J. Mannhart, Science 313, 1942 (2006).

[6] A. D. Caviglia, M. Gabay, S. Gariglio, N. Reyren, C. Cancellieri, and J.-M. Triscone, Phys. Rev. Lett. 104, 126803 (2010).

[7] M. Ben Shalom, M. Sachs, D. Rakhmilevitch, A. Palevski, and Y. Dagan, Phys. Rev. Lett. 104, 126802 (2010).

[8] A. Brinkman, M. Huijben, M. van Zalk, J. Huijben, U. Zeitler, J. Maan, W. van der Wiel, A. Rijnders, D. Blank, and J. Hilgenkamp, Nat. Mater. 6, 493 (2007).

[9] G. Cheng, M. Tomczyk, A. B. Tacla, H. Lee, S. Lu, J. P. Veazey, M. Huang, P. Irvin, S. Ryu, C.-B. Eom, A. Daley, D. Pekker, and J. Levy, Phys. Rev. X 6, 041042 (2016).

[10] C. Cen, S. Thiel, G. Hammerl, C. W. Schneider, K. E. Andersen, C. S. Hellberg, J. Mannhart, and J. Levy, Nat. Mater. 7, 298 (2008).
[11] A. Annadi, G. Cheng, H. Lee, J.-W. Lee, S. Lu, A. Tylan-Tyler, M. Briggeman, M. Tomczyk, M. Huang, D. Pekker, C.-B. Eom, P. Irvin, and J. Levy, Nano Lett. 18, 4473 (2018).

[12] M. Briggeman, M. Tomczyk, B. Tian, H. Lee, J.-W. Lee, Y. He, A. Tylan-Tyler, M. Huang, C.-B. Eom, D. Pekker, R. S. K. Mong, P. Irvin, and J. Levy, Science 367, 769 (2020).

[13] F. Bi, M. Huang, C.-W. Bark, S. Ryu, S. Lee, C.-B. Eom, P. Irvin, and J. Levy, J. Appl. Phys. 119, 025309 (2016).

[14] K. A. Brown, S. He, D. J. Eichelsdoerfer, M. Huang, I. Levy, H. Lee, S. Ryu, P. Irvin, J. Mendez-Arroyo, C.-B. Eom, C. A. Mirkin, and J. Levy, Nat. Commun. 7, 10681 (2016).

[15] J. I. Cirac and P. Zoller, Nat. Phys. 8, 264 (2012).

[16] B. Lanyon, J. Whitfield, G. Gillett, M. Goggin, M. Almeida, I. Kassal, J. Biamonte, M. Mohseni, B. Powell, M. Barbieri, A. Aspuru-Guzik, and A. White, Nat. Chem. 2, 106 (2010).

[17] I. Bloch, J. Dalibard, and S. Nascimbène, Nat. Phys. 8, 267 (2012).

[18] T. Grujic, S. R. Clark, D. Jaksch, and D. G. Angelakis, New J. Phys. 14, 103025 (2012).

[19] R. Blatt and C. F. Roos, Nat. Phys. 8, 277 (2012).

[20] A. A. Houck, H. E. Türeci, and J. Koch, Nat. Phys. 8, 292 (2012).

[21] A. Browaeys and T. Lahaye, Nat. Phys. 16, 132 (2020).

[22] M. Briggeman, J. Li, M. Huang, H. Lee, J.-W. Lee, K. Eom, C.-B. Eom, P. Irvin, and J. Levy, Sci. Adv. 6, eaba6337 (2020). 
[23] M. Briggeman, H. Lee, J.-W. Lee, K. Eom, F. Damanet, E. Mansfield, J. Li, M. Huang, A. J. Daley, C.-B. Eom, P. Irvin, and J. Levy, Nat. Phys. 17, 782 (2021).

[24] A. Manchon, H. C. Koo, J. Nitta, S. M. Frolov, and R. A. Duine, Nat. Mater. 14, 871 (2015).

[25] M. Lebrat, P. Grišins, D. Husmann, S. Häusler, L. Corman, T. Giamarchi, J.-P. Brantut, and T. Esslinger, Phys. Rev. X 8, 011053 (2018).

[26] S. Krinner, T. Esslinger, and J.-P. Brantut, J. Phys.: Condens. Matter 29, 343003 (2017).

[27] J. Dalibard, Enrico Fermi 191, 1 (2014). arXiv:1504.05520.

[28] N. Goldman, G. Juzeliūnas, P. Öhberg, and I. B. Spielman, Rep. Prog. Phys. 77, 126401 (2014).

[29] B. Farias, J. Melo, and C. Furtado, Eur. Phys. J. D 68, 77 (2014).

[30] B.-Z. Wang, Y.-H. Lu, W. Sun, S. Chen, Y. Deng, and X.-J. Liu, Phys. Rev. A 97, 011605(R) (2018).

[31] L. W. Cheuk, A. T. Sommer, Z. Hadzibabic, T. Yefsah, W. S. Bakr, and M. W. Zwierlein, Phys. Rev. Lett. 109, 095302 (2012).

[32] X.-J. Liu, M. F. Borunda, X. Liu, and J. Sinova, Phys. Rev. Lett. 102, 046402 (2009).

[33] P. Wang, Z.-Q. Yu, Z. Fu, J. Miao, L. Huang, S. Chai, H. Zhai, and J. Zhang, Phys. Rev. Lett. 109, 095301 (2012).

[34] L. Dell'Anna, G. Mazzarella, and L. Salasnich, Phys. Rev. A 84, 033633 (2011).

[35] L. Dell'Anna, G. Mazzarella, and L. Salasnich, Phys. Rev. A 86, 053632 (2012).

[36] Z. Zhong, A. Tóth, and K. Held, Phys. Rev. B 87, 161102(R) (2013).

[37] Y. Kim, R. M. Lutchyn, and C. Nayak, Phys. Rev. B 87, 245121 (2013).

[38] Note that $\alpha_{R}$ is independent of the effective masses: Their values are only important to evaluate the spin-orbit coupling between bands far from the $\Gamma$ point.

[39] R. Bistritzer, G. Khalsa, and A. H. MacDonald, Phys. Rev. B 83, 115114 (2011).

[40] A. Joshua, S. Pecker, J. Ruhman, E. Altman, and S. Ilani, Nat. Commun. 3, 1129 (2012).

[41] S. LaShell, B. A. McDougall, and E. Jensen, Phys. Rev. Lett. 77, 3419 (1996).

[42] L. Petersen and P. Hedegård, Surf. Sci. 459, 49 (2000).

[43] E. I. Rashba, Phys. Rev. B 86, 125319 (2012).

[44] C. Beenakker and H. van Houten, in Semiconductor Heterostructures and Nanostructures, Solid State Physics Vol. 44, edited by H. Ehrenreich and D. Turnbull (Academic Press, Cambridge, 1991), pp. 1-228.

[45] R. Landauer, IBM J. Res. Dev. 1, 223 (1957).

[46] G. Cheng, M. Tomczyk, S. Lu, J. Veazey, M. Huang, P. Irvin, S. Ryu, H. Lee, C.-B. Eom, C. Hellberg, and J. Levy, Nature (London) 521, 196 (2015).
[47] Y. V. Pershin, J. A. Nesteroff, and V. Privman, Phys. Rev. B 69, 121306(R) (2004).

[48] G. Shavit and Y. Oreg, SciPost Phys. 9, 051 (2020).

[49] M. Tinkham, Introduction to Superconductivity, 2nd ed. (McGraw-Hill, New York, 2004).

[50] P. G. De Gennes, Superconductivity of Metals and Alloys, 1st ed. (CRC Press, Boca Raton, 2018).

[51] V. J. Emery and S. A. Kivelson, Nature (London) 374, 434 (1995).

[52] G. Singh, A. Jouan, L. Benfatto, F. Couëdo, P. Kumar, A. Dogra, R. C. Budhani, S. Caprara, M. Grilli, E. Lesne, A. Barthélémy, M. Bibes, C. Feuillet-Palma, J. Lesueur, and N. Bergeal, Nat. Commun. 9, 407 (2018).

[53] D. L. Maslov and M. Stone, Phys. Rev. B 52, R5539 (1995).

[54] T.-C. Wei, D. Pekker, A. Rogachev, A. Bezryadin, and P. M. Goldbart, Europhys. Lett. 75, 943 (2006).

[55] M. Sigrist and K. Ueda, Rev. Mod. Phys. 63, 239 (1991).

[56] M. Alidoust and K. Halterman, J. Phys.: Condens. Matter 27, 235301 (2015).

[57] M. Alidoust and K. Halterman, New J. Phys. 17, 033001 (2015).

[58] M. Alidoust, Phys. Rev. B 101, 155123 (2020).

[59] M. Alidoust, K. Halterman, and O. T. Valls, Phys. Rev. B 92, 014508 (2015).

[60] M. Alidoust and K. Halterman, Phys. Rev. B 97, 064517 (2018).

[61] Z. Wang, X. Wang, and C. Kallin, Phys. Rev. B 101, 064507 (2020).

[62] C. M. Puetter and H.-Y. Kee, Europhys. Lett. 98, 27010 (2012).

[63] H. Q. Yuan, D. F. Agterberg, N. Hayashi, P. Badica, D. Vandervelde, K. Togano, M. Sigrist, and M. B. Salamon, Phys. Rev. Lett. 97, 017006 (2006).

[64] Y. Gindikin and V. A. Sablikov, Phys. Rev. B 98, 115137 (2018).

[65] M. Mahmoodian and A. Chaplik, JETP Lett. 107, 564 (2018).

[66] M. Leijnse and K. Flensberg, Semicond. Sci. Technol. 27, 124003 (2012).

[67] M. Mazziotti, N. Scopigno, M. Grilli, and S. Caprara, Condensed Matter 3, 37 (2018).

[68] F. Damanet, E. Mascarenhas, D. Pekker, and A. J. Daley, New J. Phys. 21, 115001 (2019).

[69] F. Damanet, E. Mascarenhas, D. Pekker, and A. J. Daley, Phys. Rev. Lett. 123, 180402 (2019).

[70] C. Laflamme, D. Yang, and P. Zoller, Phys. Rev. A 95, 043843 (2017).

[71] S. Uchino, M. Ueda, and J.-P. Brantut, Phys. Rev. A 98, 063619 (2018).

[72] C. Muldoon, L. Brandt, J. Dong, D. Stuart, E. Brainis, M. Himsworth, and A. Kuhn, New J. Phys. 14, 073051 (2012).

[73] N. V. Morrow, S. K. Dutta, and G. Raithel, Phys. Rev. Lett. 88, 093003 (2002). 